\title{
Dried fruits - brief characteristics of their nutritional values. Author's own data for dietary fibers content
}

\author{
Gyurova, Desislava Krasteva ${ }^{1}$, Enikova, Rositsa Kirilova ${ }^{2}$ \\ ${ }^{1}$ Department "Organic Analysis", National Center of Public Health and Analysis, 1431 Sofia, Bulgaria \\ ${ }^{2}$ Department "Microbiological Analysis", National Center of Public Health and Analysis, 1431 Sofia, Bulgaria
}

Email address:

d.k.gyurova@gmail.com (Gyurova D. K.), r.enikova@abv.bg (Enikova R. K.)

To cite this article:

Gyurova, Desislava Krasteva, Enikova, Rositsa Kirilova. Dried Fruits - Brief Characteristics of their Nutritional Values. Author's Own Data for Dietary Fibers Content. Journal of Food and Nutrition Sciences. Vol. 2, No. 4, 2014, pp. 105-109.

doi: $10.11648 /$ j.jfns.20140204.12

\begin{abstract}
A review of nutritional and dietetic properties of dried fruits, such as natural and concentrated sources of sugars, vitamins A, C, niacin, riboflavin and folate, potassium and trace elements of iron and copper, and of organic acids, phytonutrients with antioxidant properties was made. Many important properties of dry fruits have a low glycemic index and the content of dietary fiber, including insoluble fiber. The results of insoluble dietary fiber content by enzymatic-gravimetric method AOAC 991.42 of 18 kinds of different varieties Bulgarian dried fruits were shown. The highest content of fiber was found in hips (40\%), followed by dried quinces, pears, chokeberries, dried apples, and plums (from about $13 \%$ to over $23 \%$ ). A lower content of insoluble fiber in different varieties of cherries and sour cherries is established. The data are important for a healthy nutrition of Bulgarian population, but also for a diet-therapeutic practice, and problems related to motility of the gastrointestinal system, anemia, and atherosclerosis, cardiovascular and metabolic diseases.
\end{abstract}

Keywords: Dried Fruits, Insoluble Fibers, Glycemic Index

\section{Introduction}

Drying is the oldest and most reliable method for storing food and preserving its properties. Drying does not destroy the nutritional properties, as fibers, carbohydrates, minerals and vitamin A, are stable at dehydration. Dehydration, in its capacity of a basic method for fruit, vegetable and meat processing, has been used in household conditions since ancient times, while in industrial conditions it was implemented in the middle of the $19^{\text {th }}$ century. Drying methods [9] depend on the product type. Currently basic techniques are known - convective, pulverization (contact), drum (rotational), and lyophilization technique [1].

Drying is a very old traditional method for fruit and vegetable preservation. Most frequently dried fruits are apples, pears, apricots, grapes, plums, cherries, hips and occasionally - peaches and quinces. Numerous European populations have centuries-long traditions of using dried fruits, vegetables and herbs in traditional home diet, implementing mostly the natural convective method [7]. This method is particularly popular on the Balkans where there are beneficial climatic conditions for cultivation of various fruit varieties.
Fresh fruits and vegetables are perishable. They contain significant amounts of water that is a favorable medium for development of microorganisms and for the progress of certain biochemical processes affected by various enzymes (ferments). Thus the products perish quickly. In order to achieve their stability the water contained in them should be reduced to a certain minimum limit. For dried fruits this value should be from 18 to $23 \%$ and for vegetables - from 8 to $12 \%$. The elimination of a substantial portion of water allows the fruits and vegetables to decrease their volume and weight and transform into a "concentrated product", thus enabling their storage in ordinary conditions.

Fruits and vegetables are dried whole or sliced depending on their size. Plums, cherries, morello cherries, blueberries, blackcurrants, figs, peppers, parsley and celery leaves are dried whole. Apples, pears, quinces, peaches, apricots, carrots, onions, carrots, celery roots and leafy herbs are dried sliced. Before drying they have to be washed thoroughly and rough tissues, stones, skins, and seeds should be discharged.

The extent of drying is also important for the good qualities of dried fruits and vegetables. Overdried fruits containing lower water percentage rate are hard and cannot be eaten unless boiled; underdried ones with more humidity 
perish quickly.

The convective drying method [2] can be implemented in domestic and semi-industrial conditions - drying in the sun or in an oven at temperature not exceeding $55-60^{\circ} \mathrm{C}$ in order to preserve the best biological and nutritional properties as well as the plant pigments of the fruits and vegetables. Rotational or drum drying is implemented in industry where the mashed fruits and vegetables are spread on a heated rotating drum. They lose humidity in seconds and are scrubbed with a proper knife in the form of flakes. This is the way to produce potato [23] and maize dehydrated squash, mash and cornflakes. The pulverization contact thermal drying is applied for the production of products that are more sensitive to high temperatures such as powdered milk and eggs. This technique is also appropriate for drying fruit and vegetable juices.

The lyophilization process is the most expensive method. This technique consists of pre-freezing of the products to $-38^{\circ} \mathrm{C}$ and releasing the liquid phase by vacuum sublimation. This is the best currently implemented drying technique.

Dried fruits are among the healthiest dietary alternatives of foods containing refined sugar (sweets, candies, jams, fruit preserves) and are an excellent means to satisfy the desire to eat sweet things. Moderate fruit consumption is an excellent way to supply the necessary sugars and vitamins to the organism. Many manufacturers input fructose, naturally present in fruits, to sweeten their products.

While the fruit itself is very rich in natural sugars, many dried fruits [15] are additionally saccharified for taste modeling. Such products should rather be called "candies" and should be avoided in healthy diets. Some examples for additionally sugar coated dried fruits that should be avoided are listed below:

- Pineapple: Usually coated with refined saccharose;

- Banana chips: Usually fried and sweetened. It is recommended to consume dried bananas without added sugar.

- Blueberry: Added sweeteners are almost always applied due to the acerbity of the fruit.

- Plums: Usually the fruits are dipped in concentrated saccharose solution to improve the sour taste before drying.

- Watermelon: The combination of low content of nutrients and high sugar content is not beneficial.

Dried fruits can be purchased in bulk at most shops for healthy foods and/or pre-packed at customary food shops. It is necessary to check always the list of ingredients for added sugar or input food additives [15]. A list of traditionally consumed dried fruits by the Bulgarian population is presented below:

1. Apple. Apples are a marvelous source of fibers and contain many phytonutrients (natural plant components with nutritional value) with antioxidant effect.

2. Apricot. Drying is the healthiest way to preserve and have apricots available for consumption during all seasons. Dried apricots are a good source of fibers, vitamins $\mathrm{A}$ and $\mathrm{C}$, as well as iron.
3. Cherry. Cherries are called "the new antioxidant superfruit". Compared to other fruits they have substantially greater content of antioxidants as well as of important nutrients, such as beta-carotenes, folic acid and fibers.

4. Raisins. Raisins are often called "nature's candy". They contain low amounts of sodium and many fibers.

5. Plum. Dried plums are called prunes. Prunes are an excellent source of vitamins and contribute substantially to regulation of assimilation functions.

6. Pear. Pears are a good source of vitamin $\mathrm{C}$ and copper and are rich in dietary fibers.

7. Proper drying depends on temperature [22], low air humidity and good air circulation. Similarly to all preservation methods drying cause certain loss of some nutrients [19]. The nutrient changes occurring in the drying process involve:

Energy content: it is not altered but is concentrated in smaller mass/volume as the humidity is eliminated.

Fibers: not changed.

Vitamin A: vitamin A levels are preserved very well at implementing controlled thermal methods.

Vitamin C: pre-treatment with ascorbic acid [18] or lemon juice elevates vitamin $\mathrm{C}$ levels although certain losses are to be expected during drying.

Thiamin, riboflavin, niacin: their levels are relatively well preserved.

Minerals: their levels are relatively well preserved.

The best method to preserve the nutrients in dried foods [20] is to store the foods in a cool, dark and dry place and to consume them within one year.

Dried fruits are a very rich source of minerals and nuts are also rich in proteins. They have numerous healing properties because of the sufficient amount of nutrient components. Each dietologist recommends "a handful" of dried fruits in the diet with a view to healthy nutrition. Among the ten top health benefits [21] of dried fruits consumption are the following items:

- Pistachio, almonds and raisins are referred to as very effective in controlling cholesterol blood levels and blood circulation stimulation.

- Raisins and dates are recommended to individuals with anemia as they help in weight gaining and supply vitamins and minerals to the organism.

- Dried fruits are very adequate for treatment of cardiovascular problems.

- Almonds help in reducing the chronic coronary heart risk, affect favorably blood hemoglobin levels as well as support the hemopoiesis.

- Dates are very beneficial for cardiac health and prevent atherosclerosis, which is one of the causes for stroke and myocardial infarction.

- Prunes are a good source of potassium, vitamin A, fibers and copper and support high energy levels in the organism during the whole day.

In fact, dried fruits consumption supplies an immediate energy surge and causes improvement of the memory. 
As a whole, fresh fruits are healthy foods, good sources of vitamins, minerals, phytonutrients and dietary fibers. Fruits cultivated in gardens and farms are in most cases greater in size, sweeter and contain less dietary fibers compared to their "wild" analogues. According to Dr. Cordein dried fruits contain significantly more sugar than fresh ones. Some exceptions are avocado, lemons and limes that have low sugar content [16] and their consumption should not be restricted.

The majority of dried fruits preserves the nutrition value of the fresh ones and, together with them is included in the USA dietary guidelines [14] and the recommendations of the international health agencies. In principle, all dried fruits supply the main nutrients and a set of preventive bioactive ingredients that makes them valuable resources for improving food quality and reducing the risk for chronic diseases. The combination of nutritional value and pleasant taste has been a real prerequisite for dried fruits popularity and approval as healthy food for millenniums. They are naturally resistant to decay easy-to-store and transport and require relatively small expenditures.

\subsection{Nutritional Facts}

The main nutritional facts for them reveal:

1. Dried fruits, like fresh ones, have very low sodium content;

2. They are a source of dietary fibers and potassium;

3. Traditional dried fruits do not contain added sugar [13]. The sugar in them is in the form of glucose and fructose;

4. The drying process eliminates some of the water in the fruits, thus their natural sugars are concentrated [11]. That is why the total sugar content as well as the energy value is similar to those in fresh fruits but is in higher concentrations.

Traditional dried fruits have a low glycemic index (GI), which expresses the food impact on sugar blood levels in the organism.The value of GI reflects the capacity of the organism to assimilate carbohydrate foods (usually $50 \mathrm{~g}$ of available carbohydrates 0 compared to the individual's response to the same amount of carbohydrates, supplied by white bread or glucose. The foods are classified as high-carbohydrate (GI>70), moderate-carbohydrate (GI 56-69), or low- carbohydrate (GI0-55) [12].

Table 1 presents GI values as listed by [12]. Foods with high fiber content usually have low GI. Nevertheless it is considered that other factors also contribute to the glycemic response of the organism, namely: viscous texture of dried fruits (especially when chewed); their food matrix; available phenols and organic acids as well as the type of contained sugar (approximately $50 \%$ of fructose in traditional dried fruits) [27]. Dietary fibers [24] are found only in plant foods like fruits, vegetables, cereals and pulses. Two fiber types are distinguished - soluble and insoluble. Soluble fibers are dissolved in water and insoluble ones cannot be dissolved in water. Both types are important for health in different aspects. Cellulose, lignin, and the other hemicellulose compounds belong to the group of insoluble fibers. Skins and peels of fruits and vegetables are good sources of insoluble fibers. Most foods contain a mixture of soluble and insoluble fibers.

Many fruit types are rich in insoluble fibers [1]. They contain soluble and insoluble fibers although in some species the levels of insoluble fibers are higher. Insoluble fibers are an important part of human diet [4]. They are essential for stimulation of the gastrointestinal motility, regulation and acceleration of the intestinal passage and evacuation of intestinal content, and for reducing obstipation risk [5]. Dried fruits are an adequate source for intake of insoluble fibers. Thus, for example, $100 \mathrm{~g}$ of raisins contain more than $3 \mathrm{~g}$ of total fibers of which more than $70 \%(2.2 \mathrm{~g})$ are insoluble. The same portion of prunes contains more than $8 \mathrm{~g}$ of total fibers with some $50 \%$ of them insoluble. Traditionally the Bulgarian population consumes large amounts of dried fruits, especially in winter. A popular practice is to dry plums, apricots, apples, pears, cherries, morello cherries, hips, quinces and other fruits at home and in industrial conditions.

This fact determined the AIM of this study: to present the content of insoluble dietary fibers in some typical Bulgarian dried fruits, widely consumed by the population and to inform consumers, nutritionists and dietologists about their content with a view to compiling adequate diets and determination of nutritional benefits.

Table 1. Glycemic index of various dried fruits

\begin{tabular}{ll}
\hline Type of dried foods & Glycemic index \\
\hline Dates & 62 \\
Dried apples & 29 \\
Dried apricots & 30 \\
Dried peaches & 35 \\
Prunes & 29 \\
Figs & 61 \\
Raisins & 54 \\
\hline
\end{tabular}

\section{Material and Methods}

The study was conducted in the period 2010-2012. Eighteen market samples of Bulgarian dried fruits consumed by the Bulgarian population were analyzed for content of insoluble fibers by enzymatic-gravimetric AOAC method 991.42. Three parallel samples of each fruit were analyzed.

In the end of 2010 three samples from one and the same lots of dried fruits produced by a single manufacturer were selected and delivered to the laboratory:

- Dried white cherries I,

- Dried black cherries I,

- Dried pears I,

- Dried hips I and

- Dried chokeberry I.

In mid-2011 the same approach was applied for the selection of identical samples of the same fruits from other lots of the same manufacturer:

- Dried white cherries II,

- Dried black cherries II,

- Dried pears II, 
- $\quad$ Dried hips II and

- $\quad$ Dried chokeberry II.

The remaining samples were purchased from the same manufacturer who modified the assortment of his production and placed new types of dried fruits on the market, and the samples were also analyzed.

The samples were analyzed for content of insoluble fibers by the enzymatic-gravimetric method 991.42 immediately after the preparation of the analytical sample at the laboratory. Three parallel samples for each fruit were analyzed. The differences in the values for insoluble fibers are discussed below.

\section{Results and Discussion}

Table 2 presents the results of the tests for insoluble fibers content in dried fruits with listed standard uncertainty of the implemented method. Those data would be particularly important to evaluate the real intake of dietary fibers.

In principle dried fruits are very rich natural concentrates - sources of dietary fibers. Dried hips rank first and we could outline pears, quinces, followed by chokeberries and apples among the species with wider implementation in the diet.

The amount of insoluble fibers in drupes - cherries, morello cherries - was lower. Plums presented much greater differences, due obviously to multiple varieties.

Differences were observed in the values of insoluble fibers also in the differing between themselves types of white, black cherries, hips, pears and chokeberries when comparing the evidence for the different lots of analogous fruits harvested in two different years - 2010 and 2011. The most possible differences in the content of insoluble fibers could be explained by a complex of causes, such as fruit ripeness, cultivation and farming conditions, and climatic conditions; and last but not least - variety differences of the analyzed fruits.

Table 2. Content of insoluble fibers in dried fruits

\begin{tabular}{lll}
\hline Number & Tested samples & Content of insoluble fibers, \% \pm SU* (AOAC 991.42) \\
\hline 1 & Dried white cherries I & $5,02 \pm 0,13$ \\
2 & Dried white cherries II & $3,50 \pm 0,09$ \\
3 & Dried black cherries I & $3,94 \pm 0,10$ \\
4 & Dried black cherries II & $4,70 \pm 0,12$ \\
5 & Dried pears I & $23,30 \pm 0,59$ \\
6 & Dried pears II & $16,10 \pm 0,41$ \\
7 & Dried hips I & $49,20 \pm 1,24$ \\
8 & Dried hips II & $44,50 \pm 1,12$ \\
9 & Dried quinces & $22,80 \pm 0,57$ \\
10 & Dried mix-white cherries, apples and pears & $9,50 \pm 0,24$ \\
11 & Dried mix-black cherries, apples and prunes & $8,90 \pm 0,22$ \\
12 & Dried morello cherries & $6,90 \pm 0,17$ \\
13 & Prunes & $8,60 \pm 0,22$ \\
14 & Ground prunes & $13,86 \pm 0,35$ \\
15 & Dried apples & $14,50 \pm 0,37$ \\
16 & Dried chokeberries I & $17,70 \pm 0,45$ \\
17 & Dried chokeberries II & $23,84 \pm 0,61$ \\
18 & Dried chokeberries powder & $22,96 \pm 0,58$ \\
\hline
\end{tabular}

* SU - standard uncertainty

The content of insoluble fibers presented in Table 2 was provided by the enzymatic-gravimetric AOAC method 991.42 [3]. The gravimetric method [17] for insoluble fibers (such as cellulose) used in compiling the Bulgarian Food Composition Tables [26] obviously lists substantially lower values for the fibers in some analogous fruit types, respectively:

- For dried hips - 2,9\%

- For dried pears - 5,5\%

- For prunes - 1,5\%.

According to the Danish Food Composition Database using the enzymatic-gravimetric method, the content of fibers in prunes is $7,6 \%$; in dried apples $-8,7 \%$ and in dried hips $-43,0 \%$ [6]. According to the Norwegian Tables [25] the rate of dietary fibers in dried apples is $9,5 \%$.

The publications of Russian researchers and scientists reported insoluble fibers amounts (such as cellulose) in prunes in the range $9-30 \%$ [8], in dried apples - 48\%, and in dried pears - approximately $20 \%$ [10]. The principle of the implemented analytical method for determination of insoluble fibers was not reported.

Doubtlessly the differences in the values of insoluble fibers could be explained, together with intervariety differences of the tested fruits, with the principle of the implemented analytical method. In the particular food composition databases the application of the enzymatic-gravimetric method leads to similar results.

According to WHO recommendations the consumption of dietary fibers should be not less than $25 \mathrm{~g}$ daily. The dietologists recommend a daily intake of 30 to $50 \mathrm{~g}$ of dietary fibers. This is the optimal amount that does not harm the organism and the beneficial effect is perceived very quickly.

Certain other dried fruits are also a good source of insoluble fibers - raisins, dried apricots, figs, etc., thus presenting a challenge for further comprehensive scientific research. 


\section{Conclusion}

Dried fruits of Bulgarian origin are a significant source of insoluble dietary fibers. Hips, quinces, prunes, apples, pears and chokeberry (recently introduced and cultivated in Bulgaria) are the fruits that are the richest in this fibers type and are reasonably consumed by the population, especially in winter.

The implementation of the current enzymatic-gravimetric method enables the more objective determination of the content of insoluble dietary fibers like cellulose, hemicelluloses and lignin in dried fruits. This outlines the necessity of revising and completing the Bulgarian Food Composition Tables with more precise recent data for that type of products, adequate and necessary for the targets of dietetic therapy practice.

The improvement of the information for the specialists in dietetics and the goals of the policy focused on healthy nutrition require more comprehensive survey on the content of fibers in dried fruits and vegetables belonging to varieties cultivated in our geographic region.

\section{References}

[1] A. C. Melodie. Insoluble Fiber in Fruits, Healthy eating, 2014.

[2] A. Atanassov. Low Utilization thermo siphon heat exchangers operating conditions gas /air or gas/ liquid "Innovation for business." Applied research and technology demand in Varna, 2004, 3-4.

[3] AOAC Official Method 991.42. Insoluble Dietary Fiber in Food and Food Products. J. AOAC Int, 75, 1992, 360.

[4] D. Guyrova. Dietary fibres - an ingredient of carbohydrates in foods. Importance in human nutrition. Science and Dietetics, 2009, 2, 30-33.

[5] D. K. Gyurova. Dietary Fibers in Bulgarian Foods. Ph.D. Dissertation, National Centre of Public Health and Analysis. Bulgaria, 2013.

[6] http://www.foodcomp.dk/v7/fcdb_search.asp. Danish Food Composition Table, version 7.01, National Food Institute Technical University of Denmark, 2008.

[7] http://dieti.net/susheni-plodove, Dried fruits and vegetables, 2011.

[8] http://www.morehi.ru/suhofrukty. Dried fruits, caloric, energy value, content of vitamins and minerals in dried fruits, 2014.

[9] http://www.unileverfoodsolutions.bg/promotion/passion_for _taste/goodness_of_dry. Drying is natural and useful, 2014

[10] http://konstantinsmirnov.ru/samyie-poleznyie-suhofruktyi/, Most useful dried fruits, 2013

[11] http://ndb.nal.usda.gov. United States Department of
Agriculture. Dried Fruits, Nutrient Data Laboratory, 2011-12-19.

[12] http://www.glycemicindex.com. The Glycemic Index and GI Database, University of Sydney, 2011, retrieved 2011-12-19.

[13] http://ndb.nal.usda.gov. United States Department of Agriculture, Dietary Guidelines for Americans 2005 Appendix B. Food Sources of Selected Nutrients, 2011.

[14] http://ndb.nal.usda.gov. United States Department of Agriculture, Nutrition. MyPyramid.gov., 2011.

[15] http://www.fitday.com/fitness-articles/nutrition/healthy-eatin g/12-healthiest-dried-fruits.html. 12 Healthiest Dried Fruits, 2014.

[16] http://thepaleodiet.com/fruits-and-sugars/, Dr. Loren Cordain. The Paleo Diet ${ }^{\mathrm{TM}}$, Fruits and Sugars, Sugar Content of Fruit, 2014.

[17] ISO 5498-1981 (E) Agricultural food products determination of crude fibre content, general method, 1981.

[18] P. A. DiPersio, P. A. Kendall, M. Calicioglu, J. N. Sofos. Inactivation of Salmonella during drying and storage of apple slices treated with acidic or sodium metabisulfite, 2003

[19] P. A. DiPersio, P. A. Kendall, J.N. Sofos. Inactivation of Listeria monocytogenes during drying and storage of peach slices treated with acidic or sodium metabisulfite solutions. Food Micro., 2004, 21:641-648.

[20] P. A. DiPersio, P. A. Kendall, J.N. Sofos. Sensory evaluation of home dried fruit prepared using treatments that enhance destruction of pathogenic bacteria. J Food Quality, 2006, 29:47-64.

[21] P. Kavya. Top 10 Health Benefits of Eating Dry Fruits. $\mathrm{http}$ //listdose.com/top-10-health-benefits-of-eating-dry-fruit s-rtr/, 2014

[22] P. A. Kendall, and J. N. Sofos*. Drying Fruits, Colorado State University, 2012, 6/12, N9, 309.

[23] R. Darakchiev. Intensification of contact economizers for utilization of waste heat. Dissertation, Technical University of Sofia, 1990.

[24] Sh .Henley \& Sc. Misner. Dietary Fiber Cooperative Extension, College of Agriculture \& Life Sciences, The University of Arizona, 1999, http://cals.arizona.edu/pubs/water/az1127.html, August.

[25] The Norwegian Food Composition Table. The Norwegian Food Safety Authority, the Directorate of Health and Social Affairs and the Department of Nutrition at the University of Oslo, 2006.

[26] T. Tashev, G. Shishkov. Bulgarian Food Composition Tables, Sofia, Meditzina \& Fizkultura, 1975, 348.

[27] Y. Kim. "Raisins are a low to moderate glycemic index food with a corresponding low insulin index" Nutr Res; 2008, 28:304-308. 